\title{
IMPROVED METHODOLOGIES FOR THE CALCULATION OF CRITICAL EIGENVALUES IN SMALL SIGNAL STABILITY ANALYSIS
}

\author{
George Angelidis \\ Adam Semlyen \\ Department of Electrical and Computer Engineering \\ University of Toronto \\ Toronto, Ontario, Canada M5S 1A4
}

\begin{abstract}
This paper presents improved and new methodologies for the calculation of critical eigenvalues in the small signal stability analysis of large electric power systems. They augment the robustness and efficiency of existing methods and provide new alternatives. The procedures are implementations of Newton's method, inverse power and Rayleigh quotient iterations, equipped with implicit deflation, and restarted Amoldi with a locking mechanism and either shift-invert or semi-complex Cayley preconditioning. The various algorithms are compared and evaluated regarding convergence, performance and applicability.
\end{abstract}

Keywords: Small signal stability, selective eigenvalue analysis, shift-invert and Cayley transformations, inverse power and Rayleigh quotient iterations, Newton, Arnoldi, implicit deflation, locking.

\section{INTRODUCTION}

The assessment of the small signal stability of power systems is at present of increased significance due to their large size, heavy loading and complex controls. It is known that conventional methodologies for eigenvalue calculations (using the $\mathrm{QR}$ algorithm) are inadequate for large systems since thiey cannot take advantage of the sparsity of the network. Therefore, significant effort has been expended to perfecting partial eigenanalysis methodologies. Especially noteworthy are the contributions to focus on particular eigenvalues related to the dynamics of selected machines $[1,2]$; to achieve a selective modal analysis [3-5]; to obtain dominant eigenvalue solutions with series or parallel computers [6-8]; and to develop methodologies for obtaining solutions near specified shiftpoints close to the imaginary axis [9-11]. Particularly successful is the approach based on the Arnoldi method [10] which has been implemented in the program PEALS [12], used for the analysis of large complex systems.

The Arnoldi method is widely believed to be the most efficient approach for the identification of the set of dominant eigenvalues (i.e., of largest modulus) of an unsymmetrical matrix. However, in its application to the small signal stability analysis of large power systems, there remain two important drawbacks: first, when more than one eigenvalue is calculated, redundant operations increase the computational effort; second, since our interest is to obtain the critical (i.e., right-most) eigenvalues, several shift-points are usually necessary to make them successively dominant. In the present paper, both these problems are addressed and solved as follows. First, in the Amoldi process a locking mechanism is introduced so that, once an eigenpair has converged within the desired tolerance, it is frozen in the working set and not further updated. Second, a Cayley transformation is used as an alternative to multiple shift-points, so that all critical eigenvalues can be obtained in one sequence. A

95 SM 505-8 PWRS A paper recommended and approved by the IEE Eower system Engineering Committee of the IEEE Power Engineering Society for presentation at the 1995 IEEE/PES Summer Meeting, July 23-27, 1995, Portland, OR. Manuscript submitted December 22, 1994 ; made available for printing April 28, 1995. particular semi-complex Cayley transformation, of improved efficiency, has been used in this study.

Motivated by the results of our previous work [11], we have improved on Newton's method with implicit deflation, for the calculation of several eigenpairs. We have also applied simple and generalized Rayleigh quotient iterations as an alternative to Newton. Although these methods lack the robustness of the Arnoldi algorithm, with suitable initialization they proved to be as reliable and even faster. The initialization in our algorithms was performed by inverse power iterations.

To give a clear picture of the problems and solutions related to the computation of critical eigenvalues and to provide a background for the evaluation of the contributions described in the paper, we will first present an overview of alternative methodologies. Then details of the existing and new methods will be given with numerical results of the related comparative studies.

\section{PROBLEM FORMULATION}

The small signal stability problem in power systems can be formulated as a set of differential and algebraic equations [11]

$$
\begin{aligned}
\lambda \mathbf{x} & =\mathbf{A}^{\prime} \mathbf{x}+\mathbf{B u} \\
\mathbf{0} & =\mathbf{C} \mathbf{x}+\mathbf{D u}
\end{aligned}
$$

where $\lambda$ stands for the time derivative. In order to obtain the conventional eigenvalue problem

$$
\mathbf{A x}=\lambda \mathbf{x}
$$

the algebraic variables $\mathbf{u}$ are eliminated from (1). This however results in a dense state matrix A. Most eigenanalysis methods avoid doing this and, similarly, in the following we shall use the sparse eigenproblem formulation (1) for actual computations. For simplicity of presentation, however, we shall use form (2) in all equations and discussions.

\section{METHODOLOGIES}

\subsection{Classification}

Selective eigenanalysis methods calculate eigenvalues either individually or in a group. In the former case the eigenvalues are calculated sequentially, whereas in the latter case we have procedures that deal with subspaces. Thus, the methods can be classified as follows:

1) Sequential methods:

- power iterations

- Rayleigh quotient iterations (RQI)

- Newton

2) Subspace methods:

- $\quad$ subspace iterations (SI)

- Arnoldi

- Lanczos 
In this study we did not consider the Lanczos method since it applies to symmetrical matrices. There exists a variation of the Lanczos algorithm, unsymmetric Lanczos [13], suitable for unsymmetric matrices, but it lacks the necessary numerical properties to be practical and competitive.

\subsubsection{Sequential Methods}

These methods require a deflation process so that sequentially found eigenvalues are not repeated. In our algorithms we use an implicit deflation technique. It consists of maintaining a unitary basis for the subspace associated with the already known eigenvalues and enforcing each time the orthogonality of the currently calculated vector to this basis. The Gram-Schmidt orthogonalization algorithm [14] is used for this purpose.

Power iterations are very robust, but they converge very slowly. On the contrary, RQI and Newton converge very fast. They use, however, a moving shift-point that is updated after each iteration. This implies matrix factorizations entailing significant computational burden. Moreover, these methods lack robustness and require proper initialization. Variations to the Newton method with a single matrix factorization have been examined in [11].

\subsubsection{Subspace Methods}

These methods do not require any matrix factorization as part of the algorithm, which is a great advantage. However, they calculate the dominant eigenvalues and, since we wish to find the critical eigenvalues (those close to the imaginary axis), some initial transformation (preconditioning) is necessary [15]. This will generally imply a matrix factorization, but this is performed only once, at the beginning of the process.

In these methods, deflation is inherent because they work with subspaces. Their convergence properties are such that the first basis vectors of the subspace converge earlier than the rest $[16,17]$. Therefore, the computational efficiency can be increased by locking them after they have converged [18]. This implies that no more operations take place on this vectors until the termination of the algorithm. Locking is not to be confused with implicit deflation, described in the previous section.

\subsection{Preconditioning}

Preconditioning applies to power iterations and subspace methods. It is a transformation of matrix $\mathbf{A}$ of (2) into a matrix $\mathbf{S}$, which maps the critical eigenvalues of $\mathbf{A}$ to the dominant eigenvalues of $\mathbf{S}$, but keeps the eigenvectors unchanged. Subspace methods subsequently calculate the dominant subspace of $\mathbf{S}$. That is the subspace spanned by the dominant eigenvectors. There are several preconditioning techniques:

\section{1) Shift-invert transformation \\ 2) Cayley transformation \\ 3) Chebyshev transformation}

The Chebyshev preconditioning technique [18] is based on Chebyshev polynomials. We did not consider it in this study.

\subsubsection{Shift-Invert Transformation}

This is the simplest and most widely used transformation [19]. It uses a shift-point $s$ so that

$$
\mathrm{S}=\left(\mathrm{A}-\boldsymbol{s} \mathbf{I}^{-1}\right.
$$

where $I$ is the identity matrix. It maps the eigenvalues of $\mathbf{A}$ in the vicinity of $s$ to the dominant eigenvalues of $\mathbf{S}$. The use of this transformation with power iterations yields the well-known inversepower method [13]. This transformation is also used in each iteration of the RQI and Newton algorithms where the shift-point $s$ is updated (moving shift-point).
The advantage of this transformation is that it enlarges the relative spacing of the mapped eigenvalues in the spectrum of matrix $\mathbf{S}$, thereby improving the convergence of the algorithms that use it. The disadvantage is that in the case of a real matrix $\mathbf{A}$, a complex shift-point $s$ results in complex arithmetic. Another disadvantage, relevant to power system applications, is that in order to calculate all critical eigenvalues, one must scan the vicinity of the imaginary axis with several shift-points. An adaptive scanning algorithm can be easily devised for the selection of these shiftpoints, using the information obtained from previously used shiftpoints. Nevertheless, some redundant calculations (some eigenpairs will be recalculated) cannot be avoided unless some form of initial implicit deflation is used as well. This implies that the process starts with an initial unitary basis, corresponding to known or previously calculated eigenvectors, and is further expanded. The same technique can be effectively used to deflate uninteresting eigenvalues, like the ones at the origin. Some details with respect to this rarely discussed topic in the literature are in Appendix A.

\subsubsection{Cayley Transformation}

The Cayley transformation is a particular linear-fractional transformation (also known as Möbius transformation $[19,20]$ ) according to which

$$
\mathbf{S}=\left(\mathbf{A}-s_{1} \mathbf{I}\right)^{-1}\left(\mathbf{A}-s_{2} \mathbf{I}\right)=\mathbf{I}+\left(s_{1}-s_{2}\right)\left(\mathbf{A}-s_{1} \mathbf{I}^{-1}\right.
$$

where $s_{1}$ and $s_{2}$ are generally complex shift-points. The usual application, however, has real shift-points [21]. This is shown in Figure 1 . The vertical line $\angle$ with abscissa $\left(s_{1}+s_{2}\right) / 2$ is called the symmetry axis. The Cayley transformation maps the symmetry axis

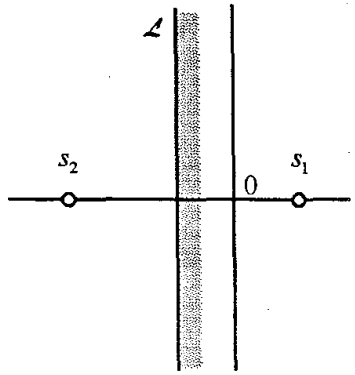

Spectral domain of $\mathbf{A}$

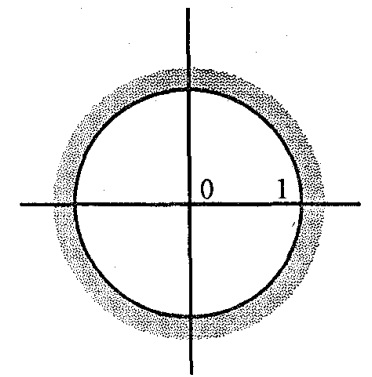

Spectral domain of $\mathbf{S}$

\section{Figure 1. Real Cayley transformation}

to the unit circle, as shown in Figure 1. The right-half plane with respect to the symmetry axis is mapped outside the unit circle. Therefore, with a suitable selection of the shift-points, the symmetry axis can be placed in such a way that the critical (right-most) eigenvalues of $\mathbf{A}$ are mapped to the dominant eigenvalues of $\mathbf{S}$.

The main advantage of the Cayley transformation is that it needs to be applied only once for the calculation of all critical eigenvalues. A single duet of shift-points and a single matrix factorization, as implied by (4), are sufficient. Additionally, since A is real, for real shift-points the calculations can be performed in real arithmetic:

Unfortunately, the advantages of the Cayley transformation do not come without shortcomings. First, the transformation reduces the relative differences in (the moduli of) the mapped eigenvalues, thereby reducing the convergence speed of selective eigenanalysis methods. Second, the complex-conjugate critical eigenvalues of $\mathbf{A}$ are mapped on the same circle. Thus, having the same modulus, they come in pairs. This counters the advantage of real arithmetic since now two real vectors have to be calculated instead of a complexconjugate pair, spanning the same subspace. Moreover, the presence of both real and complex-conjugate eigenvalues in the critical set, requires eigenanalysis algorithms that are able to alternate between 
a single-vector and a two-vector approach, depending on the type of eigenvalue. This greatly increases the complexity of the algorithms.

Because of these disadvantages of the real Cayley transformation, we relaxed the requirement for a real shift-point $s_{2}$. The result is the "semi-complex".Cayley transformation, shown in Figure 2 . The symmetry axis $\angle$ is now tilted. The advantage of this

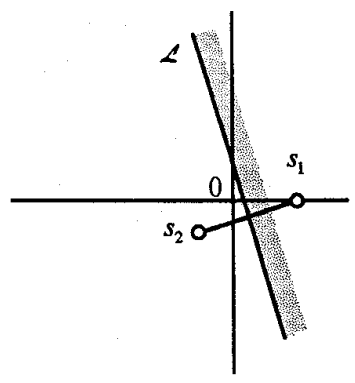

Spectral domain of $\mathbf{A}$

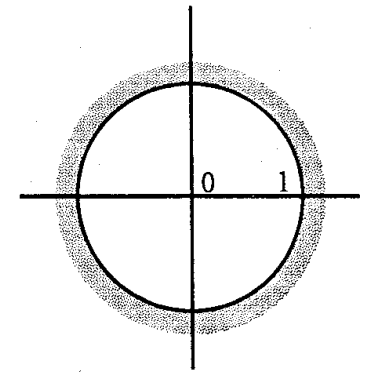

Spectral domain of $\mathbf{S}$
Figure 2. Semi-complex Cayley transformation

transformation is that it can easily discriminate between complexconjugate eigenvalues. A single-vector approach is now sufficient. Additionally, we can exclude all real eigenvalues, if we are only interested in oscillatory modes. Moreover, since $s_{1}$ remains real, the matrix factorization implied by (4) is still performed in real arithmetic.

\subsection{Solution Methods}

\subsubsection{Power Iterations}

This classical method consists of repeated matrix-vector multiplications or, in the case of inverse power iterations, forward and backward solutions

$$
(\mathrm{A}-s \Gamma) \mathbf{x}^{(r)}=\mathbf{x}^{(r-1)}
$$

where vector $\mathbf{x}$ gradually converges to the eigenvector of the eigenvalue closest to $s$. That eigenvalue is subsequently obtained by the Rayleigh quotient

$$
\lambda=\frac{\mathbf{x}^{\mathrm{H}} \mathbf{A x}}{\mathbf{x}^{\mathrm{H}} \mathbf{x}}
$$

Implicit deflation can be used in this method to calculate more eigenvalues, in the ascending order of distance to $s$. This way, the subsequent vectors do not converge to eigenvectors, but to Schur vectors instead. It is well known [17] that any matrix $\mathbf{A}$ is unitarily similar to an upper triangular matrix $\mathbf{R}$

$$
\mathbf{R}=\mathbf{Q}^{\mathrm{H}} \mathbf{A Q}
$$

where the columns of the unitary transformation matrix $\mathbf{Q}$ are the Schur vectors. They form a unitary basis for the subspace of the eigenvectors of $\mathbf{A}$. The eigenpairs can be calculated after the eigenanalysis of the projection matrix $\mathbf{R}$

$$
\mathbf{R}=\mathbf{V} \boldsymbol{\Lambda} \mathbf{V}^{-1}
$$

The eigenvalues (the elements of $\Lambda$ ) are the diagonal entries of $\mathbf{R}$ and the eigenvectors of $\mathbf{R}$ (the columns of $\mathbf{V}$ ) are obtained easily as the solution of a triangular system, subject to appropriate normalization [16]. The eigenvectors of $\mathbf{A}$ are then given by

$$
\mathbf{X}=\mathbf{Q V}
$$

The inverse power method is notorious for its slow convergence when there are many eigenvalues in the neighborhood of the shiftpoint $s[17]$.

\subsubsection{Rayleigh Quotient Iterations}

This method is an improvement on inverse power iterations. The improvement consists in updating the shift-point in (5) after each iteration, using the Rayleigh quotient (6). This moving shift-point approach requires a matrix factorization in each iteration. This increases the computational burden, but improves the convergence dramatically. It is shown in [22] that for Hermitian matrices RQI converge cubically in the neighborhood of the solution. Similarly, for non-Hermitian matrices, the use of the "generalized Rayleigh quotient"

$$
\lambda=\frac{\mathbf{y}^{\mathrm{T}} \mathbf{A x}}{\mathbf{y}^{\mathrm{T}} \mathbf{x}}
$$

also results in cubic convergence. Vector $\mathbf{y}$ in $(10)$ is an eigenvector estimate of $\mathbf{A}^{\mathrm{T}}$ (left eigenvector estimate of $\mathbf{A}$ ). The extra work needed for the calculation of $y$ amounts to an additional forward and backward substitution only, since the factors of $\left(\mathbf{A}^{\mathrm{T}}-\lambda I\right)$ are known from the factorization of $(\mathbf{A}-\lambda \mathbf{I})$. There is additional work in the deflated generalized RQI, however, due to the orthogonalization of the left Schur vector estimates Y. Moreover, the projection matrix of (7) is now given by $\left(\mathbf{Y}^{\mathrm{T}} \mathbf{X}\right)^{-1} \mathbf{Y}^{\mathrm{T}} \mathbf{A X}$ and is no longer triangular. Thus, the eigenvalue estimate cannot be simply calculated by (10), but through a complete eigenanalysis of the projection matrix.

We note that any quotient in the form:

$$
\lambda=\frac{\mathbf{w}^{\mathrm{T}} \mathbf{A x}}{\mathbf{w}^{\mathrm{T}} \mathbf{x}}
$$

where $\mathbf{w}$ is an arbitrary constant vector, can serve as an estimate of the eigenvalue that corresponds to the eigenvector estimate $\mathbf{x}$. This is so because, when $x$ converges to the exact eigenvector, then $A x \rightarrow \lambda \mathbf{x}$ and the quotient (11) converges to the exact eigenvalue. Using the quotient (11) is advantageous from a numerical point of view since the vector-matrix multiplication $\mathbf{w}^{\mathrm{T}} \mathbf{A}$ is performed only once. However, (11) is not a good estimate and results in slower convergence. It is easy to prove [22] that the Rayleigh quotient (6) is the best estimate for Hermitian matrices. Indeed, it is the leastsquares solution of (2) for $\lambda$. For non-Hermitian matrices, though, the best estimate is the generalized Rayleigh quotient (10).

We wish to point out that the cubic convergence property of the generalized Rayleigh quotient iterations has only asymptotic significance. By the time the method achieves cubic convergence, the results are usually correct within single precision tolerance [22], which is sufficient in most practical applications. Due to this reason and the additional computational burden required by the use of the generalized Rayleigh quotient, we found that simple RQI are more practical and faster. The apparent problem of increased illconditioning as $\lambda$ converges to an eigenvalue of $\mathbf{A}$, is trivial and can be handled easily [14].

\subsubsection{Newton}

This is also a moving shift-point method. For a single eigenpair, it amounts to the iterative solution of the nonlinear equation (2) for $\lambda$ and $\mathbf{x}$, subject to some normalization on $\mathbf{x}$. With the first element of $\mathbf{x}$ normalized, it takes the following form:

$$
\mathrm{J}\left[\begin{array}{c}
\Delta \lambda \\
\Delta \mathbf{x}^{\prime}
\end{array}\right]=\lambda \mathbf{x}-\mathbf{A x}
$$

where $\Delta \lambda$ and $\Delta \mathbf{x}^{\prime}$ are the updates of $\lambda$ and the unnormalized portion of $\mathbf{x}$. The Jacobian matrix $\mathbf{J}$ is the matrix $(\mathbf{A}-\lambda \mathrm{I})$ with the first column replaced by $-\mathbf{x}$. All non-incremental variables in (12) are known from the previous iteration.

Implicit deflation can be applied similarly here to calculate more than one eigenpair, but in a different way than in the methods discussed previously. It is the iterative solution of the nonlinear matrix equation 


\section{$\mathbf{A Z}=\mathbf{Z M}$}

for $\mathbf{Z}$, an invariant subspace of $\mathbf{A}$, sequentially, column after column, subject to an upper-triangular normalization of $\mathbf{Z}$ that yields an upper-triangular projection matrix $\mathbf{M}$ [11]. For the $k$ th vector $\mathbf{z}_{k}$ of the invariant subspace $\boldsymbol{Z}$, the solution takes the form:

$$
J\left[\begin{array}{c}
\Delta \mu_{k} \\
\Delta \mathbf{z}_{k}^{\prime}
\end{array}\right]=\mathbb{Z} \mu_{k}-\mathbf{A} \mathbf{z}_{k}
$$

where $\Delta \mu_{k}$ and $\Delta \mathbf{z}_{k}^{\prime}$ are the updates of $\mu_{k}$, the $k$ th column of $\mathbf{M}$, and the unnormalized portion of $\mathbf{z}_{k}$. The Jacobian matrix $\mathbf{J}$ is the matrix $\left(A-\lambda_{k} I\right)$ with the first $k$ columns replaced by $-Z$, where $\lambda_{k}$ (the $k$ th eigenvalue estimate) is the $k$ th element of $\mu_{k}$. The eigenvectors of $\mathbf{A}$ are subsequently calculated from $\mathbb{Z}$ and from the eigenvectors of the projection matrix $\mathbf{M}$, as in the deflated inverse power iteration method. The only difference here is that $\mathbb{Z}$ is not a unitary basis.

The Newton method has quadratic convergence properties, but is not robust. It is very sensitive to the initial point. It is shown in Appendix B that the Newton method for the calculation of a single eigenpair is equivalent to RQI with a particular quotient of form (11). Therefore, RQI with the quotient (6), are expected to be faster than Newton. RQI are also more robust, although they still require initialization. Nevertheless, the Newton method is still valuable as a basis for developing iterative refinement algorithms with a single matrix factorization [11]. In our algorithms, we use an adaptive approach [11] that starts with inverse power iterations and switches to Newton or RQI when a preliminary convergence is attained. If the convergence of the second stage is unsatisfactory, it is aborted and inverse power iterations proceed to calculate a better initial point. We have found [11] this algorithm to be both robust and fast. In our simulations, the adaptive part of the initialization algorithm was necessary for the single factorization variants of Newton, but not for the true Newton method. We have kept it, however, as a safeguard.

\subsubsection{Subspace Iterations}

Subspace iterations (SI), also known as simultaneous iterations, are a generalization of power iterations where instead of a single vector solution (5), a subspace solution is obtained

$$
(\mathbf{A}-s \mathbf{I}) \mathbf{X}^{(r)}=\mathbf{X}^{(r-1)}
$$

$\mathrm{X}^{(r)}$ is often orthonormalized to a unitary matrix $\mathrm{Q}$, via its $\mathrm{QR}$ decomposition [13], to maintain the linear independence of the basis vectors. Subspace iterations, although robust, suffer from the same slow convergence as power iterations. Practical implementations usually include a "Schur-Rayleigh-Ritz" (SRR) step [18], also referred to as a "Galerkin projection" step [19], which improves the convergence considerably. The SRR step consists of obtaining a better eigenvector estimate after the eigenanalysis of the projection matrix $\mathbf{R}$ of (7).

The impact of the SRR step in the algorithm is that the first vectors of the basis converge faster than the rest and the rate of convergence improves as the dimension of the subspace increases. This leads to the concepts of guard-vectors and locking. Guardvectors are extraneous vectors augmenting the subspace for the sole purpose of improving convergence. Obviously, there is a trade-off between convergence speed and computational effort. The optimal number of guard-vectors is problem-dependent. Locking, on the other hand, can be applied to vectors that meet the convergence criteria so that they are not updated any further, until the end of the process.

\subsubsection{Arnoldi}

The Arnoldi method is quite similar to subspace iterations. The difference is that the subspace is built as a unitary Krylov subspace [17]. It first starts with a single vector $x_{1}$, which is the dominant eigenvector estimate of $\mathbf{A}$. It then proceeds by calculating the second vector as $\mathbf{A x} \mathbf{x}_{1}$ and orthonormalize it with respect to $\mathbf{x}_{1}$, in what consists a Krylov step, and so on. A projection matrix $\mathbf{H}$ is automatically obtained as a by-product of the orthonormalization of the Krylov subspace, but here it is upper-Hessenberg. The original Amoldi method proceeds with the construction of the Krylow subspace until the algorithm brakes down. At that point the dominant eigenpairs of $\mathbf{A}$ can all be obtained after a complete eigenanalysis of $\mathbf{H}$ by the $\mathrm{QR}$ algorithm.

A practical implementation intentionally terminates the building of the Krylov subspace prematurely, at a specified dimension, thereby obtaining an approximate dominant subspace. The process is then restarted with a better estimate for the dominant eigenvector. Restarts reduce the performance of the method in terms of matrix-vector products and orthogonalizations (Krylov steps), but the storage requirements and the order of $\mathbf{H}$ are much less [17]. This is shown in the chart of Figure 3. The black bars correspond to the

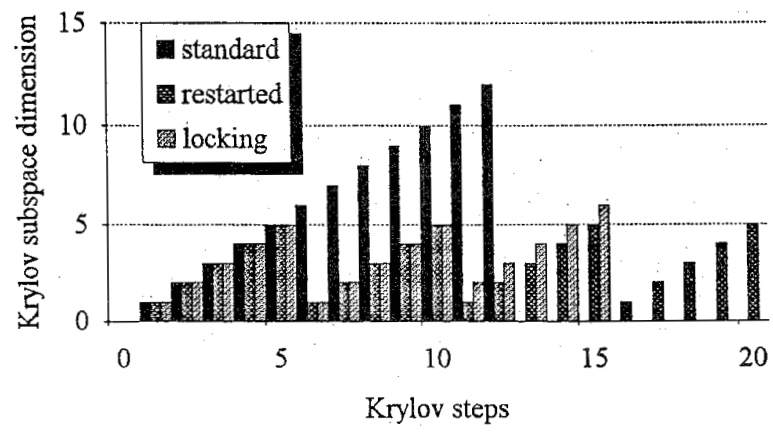

Figure 3. Amoldi method variants

standard Arnoldi algorithm. It breaks down after 12 Krylov steps, identifying the 12 most dominant eigenpairs. The dark-gray bars correspond to the restarted Arnoldi algorithm. It restarts after five Krylov steps and converges after four restarts, identifying the two most dominant eigenpairs. The convergence is here again improved as the dimension of the Krylov subspace increases. The extraneous vectors in the subspace act as guard-vectors.

As the first vectors in the Krylov subspace converge to Schur vectors, the upper-left submatrix of $\mathbf{H}$ converges to an uppertriangular matrix. Therefore, locking can also be effectively applied here, freezing the vectors of the subspace (and the corresponding columns of $\mathbf{H}$ ) as soon as they meet the convergence criteria. This is shown by the light-gray bars in the chart of Figure 3 . After two restarts, the most dominant eigenvector is identified and locked. With a constant number of guard vectors, the performance does not deteriorate for the following eigenpairs, hence this approach requires fewer restarts.

The Arnoldi method has very good convergence properties, given a sufficient number of guard vectors. Moreover, because the Krylov subspace is built sequentially, the computational effort with respect to orthonormalization is substantially less than in subspace iterations.

\section{RESULTS}

All computations were performed on an IBM PC compatible computer equipped with an Intel $486 \mathrm{DX} 2-66 \mathrm{MHz}$ processor and $16 \mathrm{MB}$ of RAM. The software package MATLAB version 4.0 was used. The algorithms were implemented as MATLAB scriptfunctions (M-files). The IEEE standard test systems of 14, 30, 57 and 118 buses [23] were analyzed. All machines were modeled with two d-axis and two q-axis circuits, and a fast exciter. Typical values were used for the machine constants according to their individual rating. All static loads were converted to constant admittances after the voltages have been obtained by power flow calculations. 
For comparing the convergence properties of the algorithms, we have selected the results tabulated in Table 1. Six algorithms were applied to calculate the four critical eigenvalues of the IEEE 14-bus test system. The initial shift point was $-1.4+\mathrm{j} 8$. It was carefully selected near the cluster so that Newton and RQI would converge to the same set of eigenvalues without special initialization. The mismatch in the last three iterations before convergence is shown. The convergence criterion was of course different in each method, but it was set so that all methods give results with $\|\mathbf{A x}-\lambda \mathbf{x}\|_{2}<10^{-6}$.

The results under the heading DIPI correspond to deflated inverse power iterations. The eigenvalues are calculated in the order shown in the Table, which is also the order of increasing distance from the shift-point. The convergence is linear and governed by the relative distance of the eigenvalues to the shift-point. It is remarkably fast for the fourth eigenvalue because the next one is quite far. DN stands for deflated Newton. It has quadratic convergence. It found the second and third eigenvalues, but then converged to a non-critical eigenvalue outside the cluster. This shows that even a good selection for the initial shift-point is not a satisfactory initialization for Newton. The next column shows the performance of Newton after initialization with DIPI. The convergence tolerance used for DIPI was $10^{-1}$. After that, Newton converged quadratically, in no more than four iterations, to the same set of critical eigenvalues and in the same order as in DIPI.

The next two columns with headings DRQI and DGRQI correspond to deflated simple and generalized Rayleigh quotient iterations. They both converge to the same set of eigenvalues as in DIPI, but in a different sequence. The second and third eigenvalues are found first (same as in DN), followed by the first and fourth. These results show that both variants of RQI are more robust than Newton since with a good initial shift-point no special initialization is necessary. In general, however, we recommend combinations like DIPI/DRQI or DIPI/DGRQI. The results show that DRQI converge quadratically whereas DGRQI converge cubically. In the latter case, even a modest convergence tolerance was enough to yield results correct to almost double arithmetic precision! However, the additional calculations in the DGRQI take a heavy toll. Even in this small problem, the execution time was $50 \%$ longer. In our simulations we found that the DIPIDRQI is by far a more practical and viable algorithm.

The last column in Table 1 with the heading RAL corresponds to the restarted Amoldi method with the locking mechanism. Four guard vectors were used to obtain the results shown in the Table. Iterations denote restarts. The method converges to the same set of eigenvalues as in DIPI and in the same order. The convergence is extremely fast, especially for the last eigenvalues. This is so because the corresponding vectors exist in the Krylov subspace as guard vectors from the beginning of the process so that they are partially converged when their turn comes. This is an advantage of subspace methods over sequential ones. The extreme occurs when more than one eigenvalues converge in one iteration (restart). In fact, with 9 guard vectors, RAL yields all four eigenvalues in a single iteration!

For comparing the performance of the algorithms with respect to execution time, we have selected the results shown in Table 2. Four algorithms were applied to calculate the four eigenvalues of the IEEE 118-bus test system, closest to the initial shift-point $-0.2+j 7$, with single precision accuracy. The total number of iterations per

Table 2. Algorithm performance

\begin{tabular}{|c||c|c|c|c|}
\hline \multirow{2}{*}{ Eigenvalue } & \multicolumn{4}{|c|}{ Iteration count } \\
\cline { 2 - 5 } & DIPI & DIPI/DN & DIPI/DRQI & RAL \\
\hline \hline$-0.2290+\mathrm{j} 7.1193$ & 22 & $6 / 3$ & $6 / 2$ & 1 \\
\hline$-0.0246+\mathrm{j} 7.1815$ & 36 & $7 / 4$ & $7 / 2$ & 1 \\
\hline$-0.2151+\mathrm{j} 7.3801$ & 121 & $10 / 5$ & $10 / 4$ & 2 \\
\hline$-0.2299+\mathrm{j} 7.4155$ & 60 & $15 / 4$ & $14 / 3$ & 1 \\
\hline \hline CPU Time [s] & 142 & 40 & 37 & 48 \\
\hline
\end{tabular}

eigenpair and the total CPU time in seconds are listed. DIPI is by far the least efficient. It requires many iterations to converge. DIPI/DRQI is slightly faster than DIPI/DN. The Arnoldi algorithm (RAL), although significantly improved since the implementation in [10] and [11], still compares unfavorably with the moving shift-point algorithms. The results shown in Table 2 are the best results for the optimal number of guard vectors, 12. It looks though that this number is excessive. Only one restart is almost enough for each eigenpair. 9 guard vectors is a more realistic number that requires more restarts and results in a CPU time of 70 seconds.

We note that these results are specific for the hardware and software used. The performance of the algorithms may be quite different on other platforms. Nevertheless, the results show that proper implementations of Newton and RQI can be serious contestants, if not clear-cut winners, over even the best implementation of the Amoldi method.

So far, the reported results with RAL were subject to an initial shift-invert transformation. It is difficult to compare this transformation with the Cayley transformation since the objective is different. Table 3 lists the critical eigenvalues of the IEEE 118-bus test system, obtained by RAL with a semi-complex Cayley transformation with shift-points $s_{1}=7$ and $s_{2}=-6-j$. Since the critical eigenvalues span a wide frequency range, it was difficult to discriminate between all complex-conjugate pairs. The transformation maps many eigenvalues near the unit-circle, hence a great number of guard vectors is required to achieve reasonable convergence. The results were obtained using 50 guard vectors. The execution time is of course $5-8$ times larger, as expected, but all

Table I. Algorithm convergence properties

\begin{tabular}{||c||c|c|c|c|c|c||}
\hline \multirow{2}{*}{ Eigenvalue } & \multicolumn{7}{|c|}{ Iteration/Mismatch } \\
\cline { 2 - 7 } & DIPI & DN & DIPIDN & DRQI & DGRQI & RAL \\
\hline \hline & $83 / 1.4 \times 10^{-6}$ & & $1 / 8.8 \times 10^{-3}$ & $4 / 4.7 \times 10^{-2}$ & $3 / 8.1 \times 10^{-2}$ & $3 / 5.9 \times 10^{-5}$ \\
$-1.5538+j 8.0997$ & $84 / 1.2 \times 10^{-6}$ & & $2 / 2.4 \times 10^{-4}$ & $5 / 5.2 \times 10^{-3}$ & $4 / 2.5 \times 10^{-2}$ & $4 / 4.0 \times 10^{-6}$ \\
& $85 / 9.8 \times 10^{-7}$ & & $3 / 2.2 \times 10^{-7}$ & $6 / 8.3 \times 10^{-5}$ & $5 / 1.9 \times 10^{-4}$ & $5 / 9.8 \times 10^{-10}$ \\
\hline \multirow{3}{*}{$-1.3570+\mathrm{j} 8.2183$} & $25 / 1.8 \times 10^{-6}$ & $4 / 1.8 \times 10^{-3}$ & $2 / 7.2 \times 10^{-3}$ & $4 / 1.0 \times 10^{-2}$ & $2 / 6.6 \times 10^{-2}$ & $1 / 3.5 \times 10^{-3}$ \\
& $26 / 1.1 \times 10^{-6}$ & $5 / 1.3 \times 10^{-5}$ & $3 / 1.6 \times 10^{-4}$ & $5 / 6.1 \times 10^{-4}$ & $3 / 6.6 \times 10^{-3}$ & $2 / 3.2 \times 10^{-6}$ \\
& $27 / 6.8 \times 10^{-7}$ & $6 / 9.2 \times 10^{-10}$ & $4 / 8.5 \times 10^{-8}$ & $6 / 3.5 \times 10^{-6}$ & $4 / 9.6 \times 10^{-6}$ & $3 / 5.8 \times 10^{-10}$ \\
\hline \multirow{3}{*}{$1.3799+\mathrm{j} 8.3632$} & $75 / 1.3 \times 10^{-6}$ & $5 / 3.0 \times 10^{-1}$ & $1 / 3.1 \times 10^{-1}$ & $3 / 4.5 \times 10^{-2}$ & $4 / 6.0 \times 10^{-1}$ & $1 / 5.2 \times 10^{-8}$ \\
& $76 / 1.1 \times 10^{-6}$ & $6 / 3.1 \times 10^{-3}$ & $2 / 5.6 \times 10^{-4}$ & $4 / 2.8 \times 10^{-3}$ & $5 / 6.3 \times 10^{-1}$ & \\
& $77 / 9.2 \times 10^{-7}$ & $7 / 3.2 \times 10^{-7}$ & $3 / 6.6 \times 10^{-8}$ & $5 / 3.0 \times 10^{-5}$ & $6 / 4.6 \times 10^{-11}$ & \\
\hline \multirow{3}{*}{$-1.6365+\mathrm{j} 8.3565$} & $5 / 2.6 \times 10^{-4}$ & & $2 / 1.4 \times 10^{-1}$ & $4 / 6.8 \times 10^{-2}$ & $4 / 5.4 \times 10^{-1}$ & $1 / 2.3 \times 10^{-10}$ \\
& $6 / 1.5 \times 10^{-5}$ & & $3 / 1.5 \times 10^{-4}$ & $5 / 4.4 \times 10^{-4}$ & $5 / 1.9 \times 10^{-3}$ & \\
& $7 / 8.1 \times 10^{-7}$ & & $4 / 1.6 \times 10^{-10}$ & $6 / 2.7 \times 10^{-8}$ & $6 / 1.1 \times 10^{-10}$ & \\
\hline
\end{tabular}


Table 3. RAL performance with Cayley transformation

\begin{tabular}{||c||c||}
\hline Eigenvalue & Iteration/Mismatch \\
\hline$-0.0899+\mathrm{j} 3.6879$ & $1 / 2.0 \times 10^{-3}$ \\
& $2 / 9.5 \times 10^{-7}$ \\
\hline$-0.0899-\mathrm{j} 3.6879$ & $1 / 2.3 \times 10^{-8}$ \\
\hline$-0.0246+\mathrm{j} 7.1815$ & $1 / 7.4 \times 10^{-7}$ \\
$-0.2418+\mathrm{j} 4.8004$ & \\
\hline$-0.2418-\mathrm{j} 4.8004$ & $1 / 1.3 \times 10^{-3}$ \\
& $2 / 9.1 \times 10^{-7}$ \\
\hline$-0.0972+\mathrm{j} 7.7662$ & $1 / 4.0 \times 10^{-6}$ \\
& $2 / 7.3 \times 10^{-7}$ \\
\hline$-0.0899+\mathrm{j} 6.2332$ & $1 / 1.0 \times 10^{-6}$ \\
& $2 / 4.6 \times 10^{-9}$ \\
\hline \hline CPU Time [s] & 391 \\
\hline
\end{tabular}

critical eigenvalues are obtained in a single run. Shift-invert transformations would require several invocations with different initial shift-points along the imaginary axis. This, plus the overhead (human or computer) of setting the shift-points, may take much longer. The Cayley transformation seems most advantageous when no a priori information is available for the eigenvalue spectrum.

\section{OVERVIEW}

Among the sequential methods, inverse power and Rayleigh quotient iterations, and Newton's method were tested and compared. The corresponding algorithms incorporated implicit deflation, a simple but very effective technique that allowed for the calculation of all eigenvalues near an initial shift-point. Inverse power iterations are very robust, albeit very slow. Their usefulness is the initialization of the other less robust methods. Newton and RQI converge quadratically with the latter being more robust and slightly faster. We have also examined the possibility of using the generalized Rayleigh quotient. We were able to confirm the cubic convergence of the generalized RQI. This method is definitely the best when only one eigenpair is to be calculated. However, in the process of calculating many eigenpairs using implicit deflation, we found that the additional computational effort with respect to the generalized Rayleigh quotient is enough to render the method inferior to simple RQI.

Among the subspace methods, restarted Arnoldi with shifinvert preconditioning was tested and compared to the sequential methods. We discounted the possibility of having subspace iterations as a competitive alternative. Simple SI is not better than deflated inverse power iterations. Of course the convergence can be dramatically improved by the introduction of a projective correction step, but this would increase the computational effort to a level higher that that of Arnoldi. Our implementation of the restarted Arnoldi method is equipped with a locking mechanism that eliminates superfluous computations that go beyond the required precision, thereby improving the efficiency and speed of the algorithm. The method is very robust and has very good convergence properties. However, its performance is heavily affected by the selection of the number of guard vectors. We note that the optimal setting for this critical parameter is problem-dependent and not known a priori.

In all our simulations with large systems, simple RQI with inverse power initialization gave the best performance. The Newton method with the same initialization came next and very close. The restarted Arnoldi with locking did not perform as well, even with the optimal number of guard vectors.

We have also investigated the possibility of using the Cayley instead of the shift-invert transformation as a preconditioner with the Arnoldi method. It is difficult to compare these two approaches directly since they have different objectives. For the objective of calculating all critical eigenvalues, the Cayley approach requires a single run whereas the shift-invert approach requires multiple runs with different shift-points. However, the former is by far more demanding in computational time than the latter. We reached the conclusion that the Cayley approach is useful when no information is available about the critical eigenvalues or when it is known that they span a wide range of frequencies. In order to improve the efficiency of the Cayley approach, we successfully applied a semicomplex Cayley transformation as opposed to the conventional real one.

\section{CONCLUSIONS}

The paper has described improved and new methodologies for the calculation of critical eigenvalues in the small signal stability analysis of large electric power systems. A wide variety of methods and techniques were applied and their performance, efficiency and applicability were evaluated and compared. They augment the robustness and efficiency of existing methods and provide new alternatives. The main findings in this study are:

- Implicit deflation is a very powerful and efficient technique. It allows methods that originally calculate a single eigenpair to be able to calculate many different eigenpairs. It can also be used to successfully deflate uninteresting or already known eigenpairs from previous runs.

- Locking is a mechanism that improves the efficiency of subspace methods where the first basis vectors converge faster than the rest. It amounts to freezing these vectors when they are within a specified tolerance.

- Restarted Arnoldi with locking is a very robust and fast method for selective eigenanalysis. Its performance is heavily influenced by the selection of the number of guard vectors.

- Newton and Rayleigh quotient iterations with implicit deflation are not robust, but when properly initialized with inverse power iterations yield robust algorithms that are faster than Amoldi.

- Semi-complex Cayley as opposed to shift-invert transformation can calculate all critical eigenvalues in a single invocation without the need for multiple shifts. The performance, however, is significantly slower due to the excessive number of guard vectors needed.

\section{ACKNOWLEDGEMENTS}

Financial support of this project by the National Sciences and Engineering Research Council of Canada and to the first author by Professor M. R. Iravani, of the University of Toronto, are gratefully acknowledged.

\section{REFERENCES}

[1] R. T. Byerly, R. J. Bennon, and D. E. Sherma, "Eigenvalue Analysis of Synchronizing Power Flow Oscillations in Large Electric Power Systems", IEEE Transactions on Power Apparatus and Systems, Vol. PAS-101, No. 1, January 1982, pp. 235-243.

[2] D. Y. Wong, G. J. Rogers, B. Porretta, and P. Kundur, "Eigenvalue Analysis of Very Large Power Systems", IEEE Transactions on Power Systems, Vol. PWRS-3, No. 2, May 1988, pp. 472-480.

[3] I. J. Pérez-Arriaga, G. C. Verghese, and F. C. Schweppe, "Selective Modal Analysis with Applications to Electric Power Systems, Part I: Heuristic Introduction", IEEE Transactions on Power Apparatus and Systems, Vol. PAS-101, No. 9, September 1982, pp. 3117-3125.

[4] G. C. Verghese, I. J. Pérez-Arriaga, and F. C. Schweppe, "Selective Modal Analysis with Applications to Electric Power Systems, Part I: The Dynamic Stability Problem", IEEE Transactions on Power Apparatus and Systems, Vol. PAS-101, No. 9, September 1982, pp. 3126-3134. 
[5] J. L. Sancha and I. J. Pérez-Arriaga, "Selective Modal Analysis of Power System Oscillatory Instability", IEEE Transactions on Power Systems, Vol. PWRS-3, No. 2, May 1988, pp. 429-438.

[6] N. Martins, "Efficient Eigenvalue and Frequency Response Methods Applied to Power System Small Signal Stability Studies", IEEE Transactions on Power Systems, Vol. PWRS-1, No. 1, Febnuary 1986, pp. 217-226.

[7] N. Martins, H. J. C. P. Pinto, and L. T. G. Lima, "Efficient Methods for Finding Transfer Function Zeros of Power Systems", IEEE Transactions on Power Systems, Vol. PWRS-7, No. 3, August 1992, pp. 1350-1361.

[8] J. M. Campagnolo, N. Martins, and D. M. Falcao, "An Efficient and Robust Eigenvalue Method for Small Signal Stability Assessment in Parallel Computers", Paper 94 SM 557-9 PWRS, presented at the IEEE/PES 1994 Summer Meeting in San Francisco, California, July 1994.

[9] A. Semlyen and L. Wang "Sequential Computation of the Complete Eigensystem for the Study Zone in Small Signal Stability Analysis of Power Systems", IEEE Transactions on Power Systems, Vol. PWRS-3, No. 2, May 1988, pp. 715-725.

[10] L. Wang and A. Semlyen, "Application of Sparse Eigenvalue Techniques to the Small Signal Stability Analysis of Large Power Systems", IEEE Transactions on Power Systems, Vol. PWRS-5, No. 2, May 1990, pp. 635-642.

[11] G. Angelidis and A. Semlyen, "Efficient Calculation of Critical Eigenvalue Clusters in the Small Signal Stability Analysis of Large Power Systems", Paper 94 SM 556-1 PWRS, presented at the IEEE/PES 1994 Summer Meeting in San Francisco, California, July 1994.

[12] P. Kundur, G. J. Rogers, D. Y. Wong, L. Wang, and M. G. Lauby, "A Comprehensive Computer Program Package for Small Signal Stability Analysis of Power Systems", IEEE Transactions on Power Systems, Vol. PWRS-S, No. 4, November 1990, pp. 1076-1083.

[13] G. H. Golub and C. F. Van Loan, Matrix Computations, 2nd edition, John Hopkins University Press, Baltimore, 1989.

[14] A Jennings and J. J. McKeown, Matrix Computation, 2nd edition, John Wiley \& Sons, New York, 1992.

[15] K. Meerbergen, "Preconditioners for Computing Right-Most Eigenvalues", Report TW 206, Department of Computing Science, Katholieke Universiteit Leuven, Belgium, March 1994.

[16] G. W. Steward, Introduction to Matrix Computations, Academic Press, New York, 1973.

[17] Y. Saad, Numerical Methods for Large Eigenvalue Problems: Theory and Algorithms, John Wiley \& Sons, New York, 1992.

[18] I. S. Duff and J. A. Scott, "Computing Selected Eigenvalues of Sparse Unsymmetric Matrices Using Subspace Iteration", ACM Transactions on Mathematical Software, Vol. 19, No. 2, June 1993, pp. 137-159.

[19] K. Meerbergen, A Spence, and D. Roose, "Shift-Invert and Cayley Transforms for the Detection of Right-Most Eigenvalues on Nonsymmetric Matrices", Revised Report TW 200, Department of Computing Science, Katholieke Universiteit Leuven, Belgium, February 1994.

[20] D. Pedoe, Geometry-A Comprehensive Course, Cambridge University Press, Cambridge, 1970.

[21] N. Uchida and T. Nagao, "A New Eigen-Analysis Method of Steady State Stability Studies for Large Power Systems: S Matrix Method", IEEE Transactions on Power Systems, Vol. PWRS-3, No. 2, May 1988, pp. 706-714.

[22] J. H. Wilkinson, The Algebraic Eigenvalue Problem, Clarendon Press, Oxford, 1965.

[23] Y. Wallach, "Section 1.6: Example Networks and Notes" in Calculations and Programs for Power System Networks, Prentice-Hall, Englewood Cliffs, New Jersey, 1986.

\section{APPENDICES}

\section{A. Eigenvalues at the Origin}

In a power system with no bus designated as an infinite bus, there is no fixed reference for the bus voltage phase angles. These angles could be coherently altered, and as long as the angular differences between buses remain the same, the operation of the system would not be affected. This degree of freedom translates to a zero eigenvalue $\lambda_{\delta}$ in the spectrum of the system state-matrix $\mathbf{A}$. It expresses the so called "common-mode of angular oscillation". The corresponding eigenvector $\mathrm{x}_{\delta}$ is a vector with unities at the entries of the angular state-variables $\delta$ and zeros elsewhere.

Since the direct speed-related attenuation in the power system dynamics is small, there exists a second eigenvalue of $\mathbf{A}$ near the origin, $\lambda_{\omega}$. The associated eigenvector $\mathbf{x}_{\omega}$ is close to $\mathbf{x}_{\delta}$ and when $\lambda_{\omega} \rightarrow \lambda_{\delta}$ then $\mathbf{x}_{\omega} \rightarrow \mathbf{x}_{\delta}$ and, at the limit, $\mathbf{A}$ becomes defective. Normally, however, these two eigenvalues are distinct and $\mathbf{x}_{\delta}$ with $\mathbf{x}_{\omega}$ determine a well-defined subspace. For its first basis vector we can take $x_{\delta}$ and for the second we may use the orthogonal projection $\mathbf{x}_{\omega}^{\prime}$ of $\mathbf{x}_{\omega}$ onto $\mathbf{x}_{\delta}$. $\mathbf{x}_{\omega}^{\prime}$ has unities at the entries of the speed statevariables $\omega$ and zeros elsewhere.

We note that numerically the eigenanalysis in the vicinity of the origin can be more accurately performed on $\mathbf{A}^{k}$, for $k=2,3, \ldots$, (which is part of a Krylov or power iteration process) instead of on A. This yields $x_{\delta}$ and $\mathbf{x}_{\omega}^{\prime}$ directly as eigenvectors.

The eigenvalues at the origin do not possess any useful information and we want to avoid their calculation. However, selective eigenanalysis methods may expend unnecessary effort by calculating them, if the shift-point is close to the origin. This can be avoided with an initial implicit deflation that includes $\mathbf{x}_{\delta}$ and $\mathbf{x}_{\omega}^{\prime}$.

\section{B. Equivalence of Newton and RQI}

Let us consider the linearization of (2) during the $r$ th iteration of the Newton method

$$
\mathbf{A} \mathbf{x}^{(r-1)}-\lambda^{(r-1)} \mathbf{x}^{(r-1)}+\mathbf{A} \Delta \mathbf{x}-\Delta \lambda \mathbf{x}^{(r-1)}-\lambda^{(r-1)} \Delta \mathbf{x}=\mathbf{0}
$$

Combining terms in (16), yields

$$
\left(\mathbf{A}-\lambda^{(r-1)} \mathbf{I}\right) \mathbf{x}^{(r)}=\Delta \lambda \mathbf{x}^{(r-1)}
$$

In the Newton method, $\mathbf{x}^{(r)}$ is obtained from $x^{(r-1)}$ subject to a normalization of its first element to unity. From (17), Newton is equivalent to an inverse power iteration where $\Delta \lambda$ is the coefficient of normalization. At the same time, $\lambda^{(r)}$ is obtained from the first of equations (17) as follows:

$$
\mathbf{a}_{1}^{\mathrm{T}} \mathbf{x}^{(r)}-\lambda^{(r-1)}=\Delta \lambda \quad \Rightarrow \quad \lambda^{(r)}=\mathbf{a}_{1}^{\mathrm{T}} \mathbf{x}^{(r)}
$$

where $a_{1}^{T}$ is the first row of matrix $A$. It can now be seen that Newton is equivalent to RQI using a particular quotient of form (11) with a constant vector $\mathbf{w}^{\mathrm{T}}=\left[\begin{array}{llll}1 & 0 & \ldots & 0\end{array}\right]$ that reduces (11) to (18).

\section{BIOGRAPHIES}

George Angelidis (Member, IEEE) was born in Athens, Greece, in 1962. He received a Ptychion degree (B.A.Sc.) from the Aristotle University of Thessaloniki in 1984, and M.A.Sc. and Ph.D. degrees from the University of Toronto in 1988 and 1992, respectively, all in Electrical Engineering. He is currently working as a Research Fellow in the Department of Electrical and Computer Engineering at the University of Toronto. His research interests are in computer applications in large-scale electric power systems.

Adam Semlyen (Fellow, IEEE) was bom and educated in Rumania where he obtained a Dipl. Ing. degree and his Ph.D. He started his career with an electric power utility and held academic positions at the Polytechnic Institute of Timisoara, Rumania. In 1969 he joined the University of Toronto where he is a professor in the Department of Electrical and Computer Engineering, emeritus since 1988. His research interests include the steady state and dynamic analysis of power systems, electromagnetic transients, and power system optimization. 


\section{Discussion}

Nelson Martins, Leonardo T.G. Lima, Hermínio J.C.P. Pinto (CEPEL - Rio de Janeiro, RJ; UFF - Niteroi, RJ; CEPEL - Rio de Janeiro, RJ, Brazil). The authors are to be congratulated for this as well as their previous contributions to the practical eigenanalysis of power systems. The following algorithms were described and assessed in this paper:

a) Newton and Rayleigh quotient iterations with implicit deflation;

b) Preconditioning by the semi-complex Cayley transformation;

c) Restarted Arnoldi with locking.

The study models used by the authors are of reduced size. This fact may have somewhat affected the results in their comparative analysis on the various methods. Can the authors comment on that?

The authors have, in previous papers, favored the use of the sequential approach over the block approach for subspace iteration $[9,10,11]$. In Section 4 the authors emphasized an advantage of the block approach: "This is an advantage of subspace methods over sequential ones ... RAL yields all four eigenvalues in a single iteration!" We were glad since we like best the block approach.

We have been working with a new class of subspace iteration methods (Refactored Bi-Iteration) incorporating multiple moving-shifts [A]. Different preconditioning strategies are used to suit specific needs, such as:

i. Computing eigenvalue clusters or a set of eigenvalues closest to several initial shifts [A].

ii. Selectively computing the set of dominant closed-loop poles of a given transfer function $F(s)[B, C]$

iii. Selectively computing the set of low-damped and unstable eigenvalues $[\mathrm{D}, \mathrm{E}]$

The Refactored Bi-Iteration algorithm is able to compute numerous eigenpairs per run, in a reduced number of iterations. The results in [A] show that 30 eigenpairs were accurately obtained in 7 iterations, for a 1200th - order matrix.

We agree with the authors that it is computationally expensive (even with locking) to work with a large interaction matrix $\mathbf{B}$, where $\mathbf{B}=\mathbf{G}^{-1} \mathbf{H}$. We are, therefore, assessing the benefits of incorporating implicit deflation for some of the previously converged eigenpairs at the block power step of the Refactored Bi-Iteration. The eigenpairs which were implicitly deflated are then not explicitly considered in the interaction matrix B. This strategy, when judiciously used, can deflate converged eigenpairs without increasing the dimension of matrix $\mathbf{B}$.
[A]J. M. Campagnolo, N. Martins and D. M. Falcão "Refactored Bi-Iteration : A High Performance Eigensolution Method for Large Power System Matrices", paper 95 SM 509-0 PWRS presented at IEEE/PES Summer Meeting, Portland, Oregon, July 1995.

[B]N. Martins, L. T. G. Lima, H. J. C. P. Pinto "Computing Dominant Poles of Very High Order Transfer Functions", paper 95 WM 191-7 PWRS presented at the 1995 IEEE Winter Power Meeting, New York, January 1995.

[C]N. Martins - "The Dominant Pole Spectrum Eigensolver", paper submitted for presentation at the IEEE/PES Winter Meeting, Baltimore, MA, January 1996.

[D]L. T. G. Lima, L. H. Bezerra, C. Tomei, N. Martins "New Methods for Fast Small-Signal Stability Assessment of Large Scale Power Systems", paper 95 WM 190-9 PWRS presented at the 1995 IEEE Winter Power Meeting, New York January 1995.

[E] N. Martins, L. T. G. Lima, H. J. C. P. Pinto, L. H. Bezerra, J. M. Campagnolo - "An Advanced Subspace Iteration Method Incorporating Multiple Moving-Shifts and Möbius Transforms", to be presented at the CERFACS Workshop on Eigenvalues, CERFACS, Toulouse, France, October 17th-20th 1995.

Manuscript received August 30, 1995.

Karl Meerbergen (K.U. Leuven, Belgium): I would like to congratulate the authors for their careful comparison of 'sequential' and 'subspace' methods applied to small signal stability analysis.

The computation of critical (i.e. rightmost) eigenvalues is a challenging practical problem. All the methods investigated by the authors compute one or several eigenvalues close to a shift point. The choice of this shift is indeed crucial for both the speed of convergence and the robustness of the methods. From the point of view of reliability, a strategy with a fixed shift near the critical zone such that no eigenvalue is favoured strongly, instead of a moving shift-point, is indeed preferred. In that sense, the combination of subspace methods with fixed shift (for robustness) and sequential methods with moving shift (for speed) is very elegant.

I have, however, two important comments to make.

1. The subspace methods are more robust than the sequential methods, but, I wish to stress the importance of good shift selection in Arnoldi and subspace iteration as well. The user should have a good idea of the critical zone in the complex plane to guarantee the calculation of the critical eigenvalues. Examples of bad shift selection are given in reference [15]. With this comment in mind, a few general hints for the selection of the initial shift for this type of application would be very useful for future use. 
2. My second remark concerns the semi-complex Cayley transform $S=\left(A-s_{1} I\right)^{-1}\left(A-s_{2} I\right)$ with $s_{1} \in \mathbb{R}$ and $s_{2} \in \mathbb{C}$. Subspace iteration always first converges to the dominant eigenvalues of $S$. The selection of $s_{2}$ is important for the inclination of the symmetry axis $\mathcal{L}$. This choice can indeed favour the convergence of the eigenvalues in the upper half plane. However, this is not true for Arnoldi's method. It is a well-known property of Arnoldi's method applied to $S$ that (in exact arithmetic) the computed eigenvalues are independent of the parameter $s_{2}$ (see [19]), so it makes no difference whether a real or complex $s_{2}$ is used. This agrees with the theoretical result that Arnoldi's method does not converge to the dominant eigenvalues of $S$ as subspace iteration does, but to the well-separated eigenvalues lying on the boundary of the spectrum. This property is often forgotten when Arnoldi's method is used.

George Angelidis and Adam Semlyen: We wish to thank the discussers for their interest in our paper and for their remarks and useful contributions. We would like to respond with the following comments.

To Dr. Meerbergen: In the application of eigenvalue calculation to the small signal stability analysis of power systems, poorly damped eigenvalues are usually of low frequency since higher frequencies entail significantly increased losses. There may however exist eigenvalues very far out on the negative real axis. This situation simplifies both the choice for a sequence of shift-points along (or close to) the imaginary axis in a Shift-Invert approach and the judicial selection of the $s_{1}$ and $s_{2}$ parameters for a Cayley transformation. In the first case, one possible strategy is to start with a shift-point $s$ at a very low frequency, say $0.1 \mathrm{~Hz}$, and then, in the process, take the next shift-point higher up, by the radius of the identified cluster (knowing that the procedure avoids finding repeatedly the same eigenvalues).

The discusser's second remark is particularly interesting and useful, if somewhat puzzling. It is based on the not sufficiently well known or recognized fact that Arnoldi's method converges to well-separated extremal eigenvalues but not necessarily to all eigenvalues in the order of their dominance, i.e. absolute value. We note that the relevant reference [19] is now published as shown below. Our motivation for choosing a complex value for $s_{2}$ (in the "semi-complex" Cayley transformation) was mainly to avoid having pairs of equal modulus (complex-conjugate) eigenvalues and we did not encounter practical difficulties since the large negative real eigenvalues were mapped quite close to the origin by the chosen transformation. With regard to the essential purpose of the semi-complex Cayley transformation, the choice of $s_{2}$ was important in our tests. Overall, the use of this new approach had a great impact both on algorithmic complexity and on the resulting computational performance.

To Dr. Martins, Prof. Lima, and Mr. Pinto: Test results always reflect particularities of the systems (and of the software and hardware) being used and we certainly agree that larger test systems are preferable. We believe however that essentially our conclusions remain valid for both smaller and larger systems. In addition, publication of the relevant experience of the discussers, certainly contributes to enrich the available knowledge on the topic of selective eigenanalysis in power systems.

Regarding the alternatives of sequential versus block approaches, we note that sequential methods allow interactive monitoring and control of the eigenvalue calculation process. Since the eigenvalues converge sequentially in the order of their distance from the original shift-point, the process can terminate when the eigenvalues of interest in that neighborhood have been obtained. Block methods have however other attractive features, like the one indicated in the paper and emphasized in the discussion.

In closing we note that reference [11] is also published; see below. In the Appendix of the present paper, in the second paragraph the sentence before the last should read: "For its first basis vector we can take $\mathbf{x}_{\delta}$ and for the second we may use the complement $\mathbf{x}_{\omega}^{\prime}$ of the orthogonal projection of $\mathbf{x}_{\omega}$ onto $\mathbf{x}_{\delta} . "$

[11]G. Angelidis and A. Semlyen, "Efficient Calculation of Critical eigenvalue Clusters in the Small Signal Stability Analysis of Large Power Systems", IEEE Transactions on Power Systems, Vol. 10, No. 1, February 1995, pp. 427-432.

[19] K. Meerbergen, A. Spence, and D. Rose, "Shift-invert and Cayley transform strategies for detection of rightmost eigenvalues of nonsymmetric matrices", BIT 34, 1994, pp. 409-423.

Manuscript received October 9, 1995. 